\title{
ASSOCIATION BETWEEN NEUTROPHIL-TO-LYMPHOCYTE RATIO AND PLATELET-TO-LYMPHOCYTE RATIO WITH 1-YEAR MORTALITY IN HEMODIALYSIS PATIENT IN WANGAYA REGIONAL GENERAL HOSPITAL
}

\author{
I PUTU YOGI SASTRAWAN, CHRISTINA PERMATA SHALIM*
}

General Practitioner, Wangaya Regional General Hospital, Denpasar, Bali, Indonesia. Email: christina.permata.s@gmail.com Received: 05 September 2019, Revised and Accepted: 08 November 2019

\section{ABSTRACT}

Objective: The aim of this study was to determine whether neutrophil-to-lymphocyte ratio (NLR) and platelet-to-lymphocyte ratio (PLR) are associated with mortality risk in patients with hemodialysis (HD).

Methods: We conducted a retrospective cohort study among regular HD patients at Wangaya Regional General Hospital. Data were collected from patients' medical records in June 2018 and followed up until May 2019.

Results: NLR and PLR were significantly associated with 1-year mortality ( $\mathrm{p}=0.047$ and $\mathrm{p}=0.009)$, with higher NLR (NLR $>2.84)$ and higher PLR $(P L R>10)$ associated with higher risk of 1-year mortality (relative risk $[R R]=3.36$ and $R R=5.19)$.

Conclusion: NLR and PLR were significantly associated with 1-year mortality in patients with HD.

Keywords: Neutrophil-to-lymphocyte ratio, Platelet-to-lymphocyte ratio, Hemodialysis, Chronic kidney disease.

(C) 2020 The Authors. Published by Innovare Academic Sciences Pvt Ltd. This is an open access article under the CC BY license (http://creativecommons. org/licenses/by/4. 0/) DOI: http://dx.doi.org/10.22159/ajpcr.2020.v13i1.35609

\section{INTRODUCTION}

Patients with chronic kidney disease (CKD) who undergo regular hemodialysis (HD) have a higher risk of mortality compared to the general population. Five-year survival rate in HD patients is mentioned at $35.8 \%$, but this value is very different in each population [1].

Neutrophil-to-lymphocyte ratio (NLR) had been studied in medical and surgical populations. It is a prognostic marker of morbidity and mortality for numerous conditions including cardiovascular disease (CVD), oncology, critical care medicine, liver disease, general surgery, and vascular surgery. It has been examined as a novel measure of inflammation in distinct populations and has been showed to have prognostic and predictive values, especially in those with systemic inflammation [2]. It was recently found to be associated with all-cause mortality in HD patients. Many patients with CKD have increased serum levels of inflammatory mediators including C-reactive protein (CRP), tumor necrosis factor- $\alpha$, interleukin- 6 , and pentraxin-3. Monitoring CRP level is still not routine in many dialysis centers, other most of which are time consuming and expensive. The fact that NLR is simply calculated from easily available complete blood count makes it an interesting biomarker in assessing and detecting inflammatory conditions in dialysis patients [2]. Of note, very few studies have addressed the relationship between NLR and kidney disease progression in patients with CKD [3].

The same with NLR, the platelet-to-lymphocyte ratio (PLR) has been introduced as a potential marker of inflammation in CVD and tumors which are also inflammation-related diseases. A positive association between a high PLR and a poor prognosis for these diseases has been reported [4]. However, just few studies had explored the prognostic effect and renal outcomes of the PLR in CKD patients with regular HD.

\section{METHODS}

Study design and data collection

We conducted a retrospective cohort study among regular HD patients at HD center in Wangaya Regional General Hospital. Data were collected from patients' medical records in June 2018 and followed up until May 2019. Baseline data that we collected from patients' medical records in June 2018 were identity, age, sex, platelet count, neutrophil count, and lymphocyte count. Then, we followed up whether the patient was still alive or not in May 2019. Patients who did not have complete data were excluded from this study.

\section{Definition of NLR}

NLR was calculated as the ratio of the neutrophils and lymphocytes that obtained from the same laboratory result in June 2018, we collected from patients' medical records.

Some studies used definite NLR cutoff points (e.g., NLR $\geq 2.5$, NLR $\geq 2.7$, $N L R \geq 3, N L R \geq 4$, and others used $N L R \geq 5$ ). In this study, patients' NLR was separated into two groups according to median value of NLR ( NLR>2.84 and $N L R \leq 2.84)$.

\section{Definition of PLR}

PLR was calculated as the ratio of the platelets and lymphocytes that obtained from the same laboratory result in June 2018, we collected from

Table 1: Baseline characteristics

\begin{tabular}{ll}
\hline Characteristics & $\begin{array}{l}\text { Number (\%) or median } \\
\text { (interquartile range) }\end{array}$ \\
\hline Age & $51(47-58.5)$ \\
Sex & $36(67.9)$ \\
Male & $17(32.1)$ \\
Female & $9.7(8.9-10.5)$ \\
Hemoglobin (g/dL) & $2.84(2.27-3.82)$ \\
NLR & $10(7.2-12.86)$ \\
PLR & $41(77.4)$ \\
Outcome after 1-year follow-up & $12(22.6)$ \\
$\quad$ Alive & \\
Die & \\
\hline
\end{tabular}

NLR: Neutrophil-to-lymphocyte ratio, PLR: Platelet-to-lymphocyte ratio 
Table 2: Bivariate analysis of NLR and PLR with patient's 1-year mortality

\begin{tabular}{|c|c|c|c|c|c|}
\hline \multirow[t]{2}{*}{ Characteristics } & \multirow[t]{2}{*}{ Total (\%) } & \multicolumn{2}{|c|}{ Outcome after 1 year $(\%)$} & \multirow[t]{2}{*}{ p value } & \multirow[t]{2}{*}{$\mathbf{R R}$} \\
\hline & & Died & Alive & & \\
\hline \multicolumn{6}{|l|}{ NLR } \\
\hline$\leq 2.84$ & $28(52.8)$ & $3(25)$ & $25(61)$ & & \\
\hline \multicolumn{6}{|l|}{ PLR } \\
\hline$>10$ & $26(49.1)$ & $10(83.3)$ & $16(39)$ & 0.009 & 5.19 \\
\hline$\leq 10$ & $27(50.9)$ & $2(16.7)$ & $25(61)$ & & \\
\hline
\end{tabular}

NLR: Neutrophil-to-lymphocyte ratio, PLR: Platelet-to-lymphocyte ratio

patients' medical records. In this study, patients' PLR was separated into two groups according to median value of PLR (PLR>10 and PLR $\leq 10)$.

\section{Data analysis}

We performed analysis using SPSS 20.0. Descriptive statistics for categorical data were obtained using frequency distributions while for continuous data measures of central tendency and spread, based on normally distributed data, were used. Chi-square test was used for bivariate analysis. $\mathrm{p}<0.05$ was considered statistically significant.

\section{RESULTS}

A total of 53 regular HD patients' data in Wangaya Regional General Hospital on June 2018 were collected. Among them, $67.9 \%$ of the patients were male and $32.1 \%$ were female, with a median age of 51 years old. The median NLR was $2.83(2.27-3.82)$ and the median PLR was 10 (7.2-12.86). Baseline characteristics of all study participants are described in Table 1.

After 1 year of follow-up, 12 patients died (22.6\%) and 41 patients remained alive (77.4\%). NLR and PLR were significantly associated with 1-year mortality $(\mathrm{p}=0.047$ and $\mathrm{p}=0.009)$, with higher NLR $(N L R>2.84)$ and higher PLR (PLR>10) associated with higher risk of 1 -year mortality (relative risk $[R R]=3.36$ and $R R=5.19$ ) (Table 2).

\section{DISCUSSION}

This study aimed to find out whether there is a correlation between NLR and PLR values with 1-year mortality of patients undergoing HD. In this study, we found a significant relationship between NLR with 1-year mortality of HD patients $(\mathrm{p}=0.047)$ and higher NLR $(\mathrm{NLR}>2.84)$ associated with higher risk of 1-year mortality $(\mathrm{RR}=3.36)$.

The main mechanism underlying the relationship between NLR and these poor outcomes was thought to be an increase in chronic inflammation in CKD patients that probably related to higher NLR. The study by Amdur et al. showed that elevated plasma level of various inflammatory biomarkers was associated with rapid loss of kidney function in patients with CKD [5]. Meanwhile, even a modest decrease in kidney function is also associated with increased risk for cardiovascular events and death [6].

Yoshitomi et al., in their study, demonstrated that NLR was correlated with higher CRP levels, lower eGFR, and the presence of ischemic heart disease (IHD). These findings suggested that high NLR reflects chronic inflammation and poorer renal outcomes [3]. The presence of IHD also might be associated with poorer prognosis in CKD patients because CVD was known to be the most common causes of death in patients' with declined kidney function [7].

In this study, we also obtained a significant correlation between PLR with 1-year mortality of HD patients $(\mathrm{p}=0.009)$. Mechanisms behind this was likely similar with what we assume underlie NLR and 1 year mortality relationship, with chronic inflammation become the main factor. This association was shown from several previous study findings that showed that high PLR is related to the presence of other conditions that had high inflammation process $[8,9]$.
It has been suggested that the PLR could be a good marker of inflammation in the population with CKD [10]. Several studies also showed a positive relationship between PLR values and disease severity. In a study of 2563 patients, Velibey et al. demonstrated that increased PLR is independently associated with a greater risk of contrast-induced acute kidney injury (AKI) in patients undergoing primary percutaneous coronary intervention [11]. Although this study took samples from the AKI patients, both AKI and CKD are associated with local and systemic inflammation, so we thought its result still relevant with our study finding.

Zhou et al. in their study showed that PLR is independently associated with CAD severity and long-term major adverse CVD events. This might explain higher incidence of mortality in our patients with higher PLR [12].

Several studies even showed that PLR has better predictive value for diagnosing inflammation than NLR in HD patients $[13,14]$. This was in line with our findings that showed that PLR had a statistically more significant relationship to 1-year mortality than NLR ( $p=0.009$ and $\mathrm{p}=0.047$, respectively).

The median PLR value in this study is relatively small. This is probably due to the small number of samples, but we found that higher PLR value $(P L R>10)$ associated with higher risk of 1-year mortality (RR=5.19).

In relation to the PLR, there are not enough data to define a definitive cutoff point. However, a recent study with over 100,000 patients on hemodialysis has established a J-Curve pattern for death: Values $<100$ and $>300$ would have higher mortality rates than those in the range of 100-150. The same result was found by Zheng et al. Their study found a U-shaped relationship between the PLR and 30-day and 90-day mortality, with both low and high PLRs were associated with increased all-cause mortality [4].

The present study had some limitations. The study subjects were recruited in single regional hospital. Thus, the selection of patients was limited and the sample size was relatively small. A larger cohort study will be needed to avoid study bias.

\section{CONCLUSION}

NLR and PLR were significantly associated with 1-year mortality in patients with regular HD.

\section{AUTHORS' CONTRIBUTIONS}

All authors have contributed equally in developing the concept of the study, data collection, data analysis, and drafting the manuscript.

\section{CONFLICTS OF INTEREST}

The authors declare that there are no conflicts of interest regarding the publication of this article.

\section{REFERENCES}

1. Mailloux LU, Henrich WL. Patient Survival and Maintenance Dialysis; 2014. Available from: http://www.uptodate.com/subscribers/tutorial/ 
index.asp. [Last accessed on 2011 Mar 20].

2. Pineault J, Lamarche C, Bell R, Lafrance JP, Ouellet G, Leblanc M, et al. Association of neutrophil-to-lymphocyte ratio with inflammation and erythropoietin resistance in chronic dialysis patients. Can J Kidney Heal Dis 2017;4:1-7.

3. Yoshitomi R, Nakayama M, Sakoh T, Fukui A, Katafuchi E, Seki M, et al. High neutrophil/lymphocyte ratio is associated with poor renal outcomes in Japanese patients with chronic kidney disease. Ren Fail 2019;41:238-43.

4. Zheng CF, Liu WY, Zeng FF, Zheng MH, Shi HY, Zhou Y, et al. Prognostic value of platelet-to-lymphocyte ratios among critically ill patients with acute kidney injury. Crit Care 2017;21:238.

5. Amdur RL, Feldman HI, Gupta J, Yang W, Kanetsky P, Shlipak M, et al. Inflammation and progression of CKD: The CRIC study. Clin J Am Soc Nephrol 2016;11:1546-56.

6. Guo Y, Cui L, Ye P, Li J, Wu S, Luo Y. Change of kidney function is associated with all-cause mortality and cardiovascular diseases: Results from the kailuan study. J Am Heart Assoc 2018;7:e010596.

7. Thompson S, James M, Wiebe N, Hemmelgarn B, Manns B, Klarenbach S, et al. Cause of death in patients with reduced kidney function. J Am Soc Nephrol 2015;26:2504-11.

8. Gary T, Pichler M, Belaj K, Hafner F, Gerger A, Froehlich H, et al. Platelet-to-lymphocyte ratio: A novel marker for critical limb ischemia in peripheral arterial occlusive disease patients. PLoS One 2013;8:e67688.
9. Badora-Rybicka A, Nowara E, Starzyczny-Słota D. Neutrophil-tolymphocyte ratio and platelet-to-lymphocyte ratio before chemotherapy as potential prognostic factors in patients with newly diagnosed epithelial ovarian cancer. ESMO Open 2016:e000039.

10. Valga F, Monzón T, Henriquez F, Antón-Pérez G. Neutrophil-tolymphocyte and platelet-to-lymphocyte ratios as biological markers of interest in kidney disease. Nefrologa 2019;39:243-9.

11. Velibey Y, Oz A, Tanik O, Guvenc TS, Kalenderoglu K, Gumusdag A, et al. Platelet-to-lymphocyte ratio predicts contrast-induced acute kidney injury in patients with st-segment elevation myocardial infarction undergoing primary percutaneous coronary intervention. Angiology 2017;68:419-27.

12. Zhou D, Wang G, Fan Y, Wan Z, Liu X. platelet to lymphocyte ratio is associated with the severity of coronary artery disease and clinical outcomes of percutaneous coronary intervention in the chinese han population. Exp Ther Med 2017;13:731-8.

13. Valencia VC, de la Cruz CO, Rodríguez OM, Castellanos SG, Rangel FA, Sandoval ME. Inflammation in hemodialysis and their correlation with neutrophi-lymphocite ratio and platelet-lymphocyte ratio. Nefrologa 2017;37:554-6.

14. Turkmen K, Erdur FM, Ozcicek F, Ozcicek A, Akbas EM, Ozbicer A, et al. Platelet-to-lymphocyte ratio better predicts inflammation than neutrophil-to-lymphocyte ratio in end-stage renal disease patients. Hemodial Int 2013;17:391-6. 